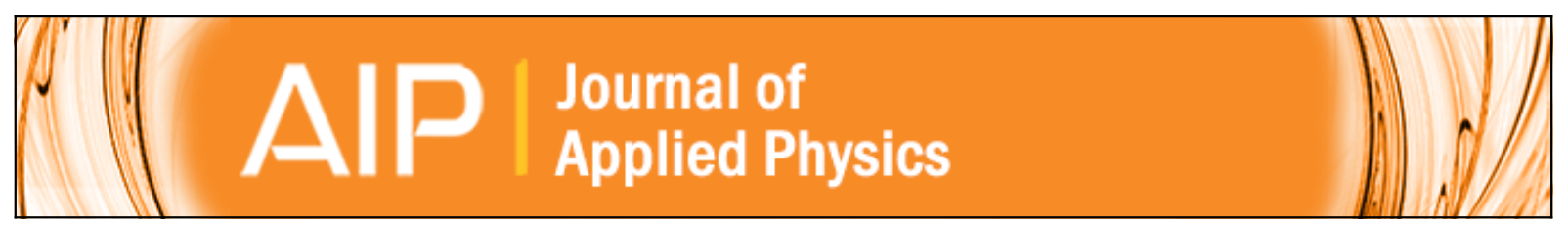

\title{
Thermal hysteresis of local instabilities in paraelectric phase of PbZr0.96Sn0.04 03 single crystals
}

Irena Jankowska-Sumara, Krzysztof Szot, Andrzej Majchrowski, and Krystian Roleder

Citation: Journal of Applied Physics 113, 187209 (2013); doi: 10.1063/1.4801980

View online: http://dx.doi.org/10.1063/1.4801980

View Table of Contents: http://scitation.aip.org/content/aip/journal/jap/113/18?ver=pdfcov

Published by the AIP Publishing

\section{Articles you may be interested in}

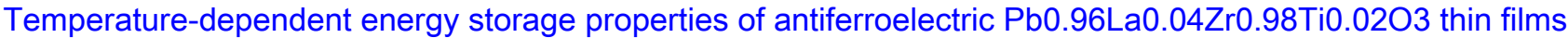
Appl. Phys. Lett. 104, 263902 (2014); 10.1063/1.4887066

Electric field induced metastable ferroelectric phase and its behavior in (Pb, La) $(\mathrm{Zr}, \mathrm{Sn}, \mathrm{Ti}) \mathrm{O} 3$ antiferroelectric single crystal near morphotropic phase boundary

Appl. Phys. Lett. 104, 052912 (2014); 10.1063/1.4864317

Effect of electrode materials on the scaling behavior of energy density in $\mathrm{Pb}(\mathrm{Zr0} 0.96 \mathrm{Ti0} 03) \mathrm{Nb0.0103}$ antiferroelectric films

Appl. Phys. Lett. 101, 112905 (2012); 10.1063/1.4752726

Enhanced antiferroelectric and electric-induced pyroelectric properties of Mn-substitued

(Pb0.832Ba0.138La0.02)(Zr0.7Ti0.05Sn0.25)O3 ceramics

Appl. Phys. Lett. 101, 062906 (2012); 10.1063/1.4744948

Phase transition behaviors of $\mathrm{PbZr} 1-\mathrm{x} \mathrm{Ti} \times \mathrm{O} 3$ single crystals as revealed by elastic anomalies and central peaks

Appl. Phys. Lett. 100, 082903 (2012); 10.1063/1.3688050

Frustrated by

old technology?

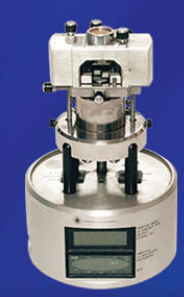

Is your AFM dead

and can't be repaired?

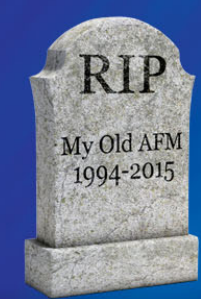

Sick of bad customer support?

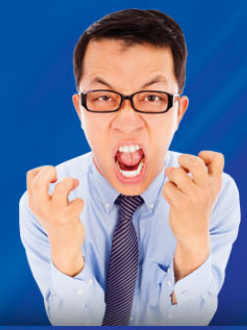

It is time to upgrade your AFM Minimum \$20,000 trade-in discount for purchases before August 31st

Asylum Research is today's technology leader in AFM 


\title{
Thermal hysteresis of local instabilities in paraelectric phase of $\mathrm{PbZr}_{0.96} \mathrm{Sn}_{0.04} \mathrm{O}_{3}$ single crystals
}

\author{
Irena Jankowska-Sumara, ${ }^{1,2}$ Krzysztof Szot, ${ }^{2,3}$ Andrzej Majchrowski, ${ }^{4}$ \\ and Krystian Roleder ${ }^{3}$ \\ ${ }^{1}$ Institute of Physics, Pedagogical University of Cracow, ul. Podchorażych 2, Kraków, Poland \\ ${ }^{2}$ Peter Grünberg Institute, Forchungzentrum Jülich, D-52425 Jülich, Germany \\ ${ }^{3}$ Institute of Physics, University of Silesia, ul. Uniwersytecka 4, Katowice, Poland \\ ${ }^{4}$ Institute of Applied Physics, Military University of Technology, ul. Kaliskiego 2, Warszawa, Poland
}

(Received 30 September 2012; accepted 8 February 2013; published online 8 May 2013)

\begin{abstract}
Investigations of local electromechanical properties for $\mathrm{PbZr}_{1-x} \mathrm{Sn}_{x} \mathrm{O}_{3}$ single crystals are a way to understand better the phase transition mechanisms in oxide perovskites with antiferroelectric phase transition. The investigations of local electromechanical studies were performed by the PEFM. The piezoelectric activity in nano-scale and weak birefringence observed in micro-scale have been found in the paraelectric phase and are a proof for theoretically predicted local instability above $T_{\mathrm{C}}$ [A. Bussmann-Holder et al., Phys. Rev. B 79, 184111 (2009)]. Origin of these instabilities is analysed taking into account non-homogeneous distribution of Sn in crystal lattice, presence of extended defects and other lattice inhomogeneities. Temperature dependences and thermal hysteresis of piezo- and electrostrictive responses were compared with bulk properties. (C) 2013 AIP Publishing LLC [http://dx.doi.org/10.1063/1.4801980]
\end{abstract}

\section{INTRODUCTION}

Lead zirconate $\mathrm{PbZrO}_{3}$ was the first compound identified as an antiferroelectric (AFE). In low-temperature phase $\left(T<T_{\mathrm{C}}=505 \mathrm{~K}\right), \mathrm{PbZrO}_{3}$ crystallizes in an orthorhombic Pbam symmetry. ${ }^{2}$ At high temperature, $\mathrm{PbZrO}_{3}$ displays the prototype paraelectric (PE) Pm3m cubic perovskite structure, in which existence of polar clusters, ${ }^{3,4}$ associated with a harmonic lead-ions hopping, was reported. Pronounced dielectric permittivity maximum reaching value of several thousand, ${ }^{5-7}$ and its thermal hysteresis observed around $T_{\mathrm{C}}=505 \mathrm{~K}$, revealed the first-order phase transition. In between the AFE and PE structures, an intermediate ferroelectric phase of rhombohedral symmetry may appear. ${ }^{8-11}$ Temperature range of its existence varies, and origin of this intermediate ferroelectricity is not known yet. Here, we would like to mention two attempts of interpretation: (i) oxygen octahedra rotation mode proposed in Ref. 11 as that responsible for transient phase appearance and (ii) presence of defect with define concentration ${ }^{12}$ that induces the ferroelectric properties below $T_{\mathrm{C}}$.

Therefore, modification, in a controlled way, of the delicate balance between the ferroelectricity and antiferroelectricity by changing crystal composition is a strong motivation to study $\mathrm{PbZrO}_{3}$ single crystals properties. Different substitutions influence differently the intermediate ferroelectric phase stability. The most intensively studied are $\mathrm{Ti}$ or $\mathrm{Sn}$ ions in place of $\mathrm{Zr}$ and $\mathrm{Nb}, \mathrm{Ba}$ and $\mathrm{La}$ in place of $\mathrm{Pb}$ ions. ${ }^{13}$

In our paper, ${ }^{14}$ it was established that increase of Sn concentration in $\mathrm{PbZrO}_{3}$ leads to the enhancement of covalent interactions between atoms. Strong covalent interactions modify lattice distortions; and from this point of view, the phase diagram for the $\mathrm{PbZr}_{1-x} \mathrm{Sn}_{x} \mathrm{O}_{3}$ (PZS) solid solutions with $0<x<0.35$ was investigated in a wide temperature range.
It was found that for small amount of $\mathrm{Sn}$ in $\mathrm{PbZr}_{1-x} \mathrm{Sn}_{x} \mathrm{O}_{3}$ solutions $(x \leq 0.2)$, ferroelectricity is squeezed out and sample undergoes transition to antiferroelectric state only.

In our earlier works, ${ }^{15,16}$ temperature dependence of bulk electrostriction and birefringence measured above $T_{\mathrm{C}}$ for $\mathrm{PbZr}_{1-x} \mathrm{Sn}_{x} \mathrm{O}_{3}$ with such antiferroelectric transition has shown an anomalous behavior. The characteristic feature was a departure of the temperature dependence of the electrostrictive coefficient $M(\mathrm{~T})$ from the one expected for pure centrosymmetric paraelectric phase. Another feature was the presence of birefringence forbidden by symmetry of paraelectric phase and existence of a local $\Delta n(\mathrm{~T})$ maximum in similar temperature range. That is why we decided to investigate temperature dependences of local piezoelectric and electrostrictive properties of single PZS crystals by means of piezo- and electrostrictive response force microscopy (PEFM) method and compare them with macroscopic (bulk) responses.

\section{ELECTROSTRICTION AND PIEZOELECTRIC EFFECT}

The electrostriction as well as piezoelectricity are most often described by the coefficients $M$ and $d$, appearing in equations defining these phenomena. The strain $\eta$ of the sample placed in an electric field $E$ can be described by the following equation:

$$
\eta_{j k}=d_{i j k} E_{i}+M_{i l j k} E_{i} E_{l}
$$

where $\eta_{\mathrm{jk}}$ is the strain tensor, and $E_{\mathrm{i}}$ and $E_{1}$ are the components of electric field vector. The first term describes the linear piezoelectric effect, which disappears if centre of symmetry is present, and $d_{\mathrm{ijk}}$ is called piezoelectric modulus. The second 
term describes the electrostrictive effect, in which coefficient $M_{\mathrm{iljk}}$ creates fourth order tensor symmetrical in relation to $i$ and $l$ and also $j$ and $k$. Electrostrictive effect appears independently on crystal symmetry and obeys the dependence of the strain on second power electric field. In crystals with cubic symmetry (as expected in ferroelectrics above $T_{\mathrm{c}}$ ), the strain is of pure electrostrictive character and is written as

$$
\eta_{j k}=M_{i l j k} E_{i} E_{l}
$$

In this paper, longitudinal $\eta_{1}$ and transverse $\eta_{2}$ macroscopic electrostrictive strains, in Voigt notation,

$$
\begin{aligned}
& \eta_{1}=M_{11} E_{1}^{2} \\
& \eta_{2}=M_{21} E_{1}^{2}
\end{aligned}
$$

will be considered. While these equations are valid for macroscopic strain in single crystals, in the case of PEFM method in nanoscale-because of non-homogenous distribution of the electric field under tip-description of the piezoelectric and electrostrictive strain with the use of Eq. (3) is not correct.

\section{MACROSCOPIC (BULK) MEASUREMENTS}

The measurements were made on crystals grown by spontaneous crystallization (flux method). Details of growing process are described in Ref. 14. Among the $\mathrm{PbZr}_{1-x} \mathrm{Sn}_{x} \mathrm{O}_{3}$ solid solutions, a crystal with $x=0.04$ was chosen for experiments. For this sample, the precise differential scanning calorimetry (DSC) measurements (Fig. 1(a)) and $\varepsilon^{\prime}(T)$ run (Fig. 1(b)) have clearly revealed two phase transformations to low temperature phase, with transient antiferroelectric phase below $T_{\mathrm{c}}$.

It is worth noting that above $T_{\mathrm{C}}$ weak but visible over the background birefringence has unexpectedly been detected (Figure 2). These very small changes in birefringence, of the order of $10^{-6}$, were determined by the rotating analyzing method described in Ref. 17. This birefringence, with a thermal hysteresis, was observed in temperature range of about $50 \mathrm{~K}$ above transition to the paraelectric phase. This experimental result indicates (local) breaking symmetry effect, most probably leading to existence of regions of polar character. The existence of such regions in perovskite ferroelectrics has already been addressed and has been interpreted in terms of pre-transitional effects. ${ }^{1,18}$ Mentioned above, temperature range $\Delta T=50 \mathrm{~K}$ with the non-zero birefringence is in good agreement with the theoretical predictions by Bussmann-Holder. ${ }^{1,19}$ According to this reports, the $\Delta T=1.1 * T_{\mathrm{C}}-T_{\mathrm{C}}$ and, in our case, it is equal to $1.1 *$ $(230+273.16)]-(230+273.16)$ what gives $50.3 \mathrm{~K}$.

In our previous papers, we have already presented abnormal behavior of electrostrictive effect in the paraelectric phase in PZS single crystals with different Sn concentrations. ${ }^{16}$ Deviations of the $M(T)$ run from classical one was the stronger the higher was $\mathrm{Sn}$ concentration. We believe that origin of these deviations came from inhomogenously distributed (disordered) Sn ions, substituting $\mathrm{Zr}$ centrally placed in the oxygen octahedron. However, those experiments have been performed on heating only. Thus, this
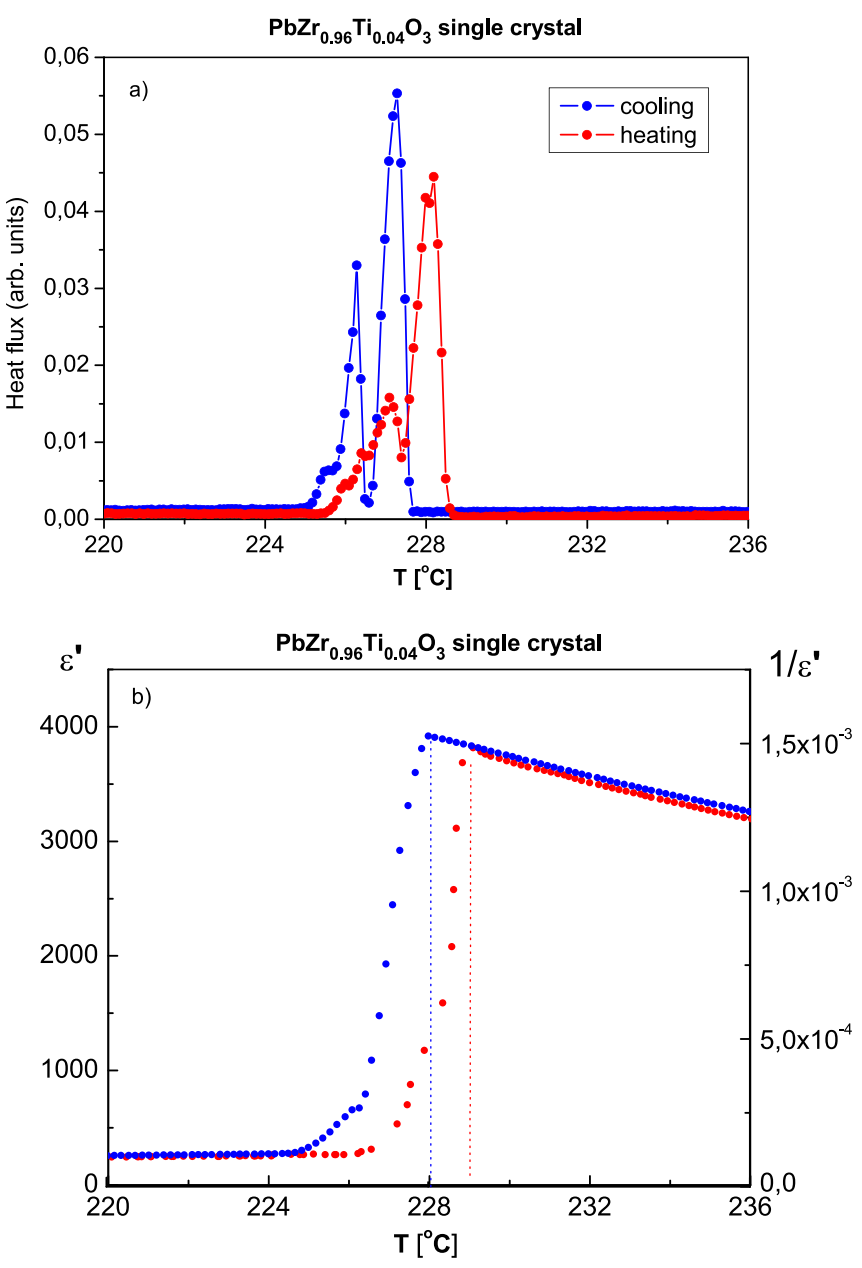

FIG. 1. DSC (a) and $\varepsilon^{\prime}(\mathrm{T})$ (b) measurements revealing phase transitions in $\mathrm{PbZr}_{0.96} \mathrm{Sn}_{0.04} \mathrm{O}_{3}$ single crystal. Lower peak in DSC and a hump anomaly in $\varepsilon^{\prime}(\mathrm{T})$ run close to $\mathrm{T}_{\mathrm{c}}$ point to existence of transient phase.

motivated us to re-measure the electrostrictive behavior for chosen crystal on heating and cooling. Hence, the electrostrictive strain $\eta$ was tentatively measured with very sensitive capacitance method. ${ }^{20}$ As seen in Figure 3, clear thermal hysteresis of the $M_{11}(\mathrm{~T})$ runs can be observed and range of this hysteresis is well correlated with the temperature range, where non-zero birefringence exists. However, in contrary to

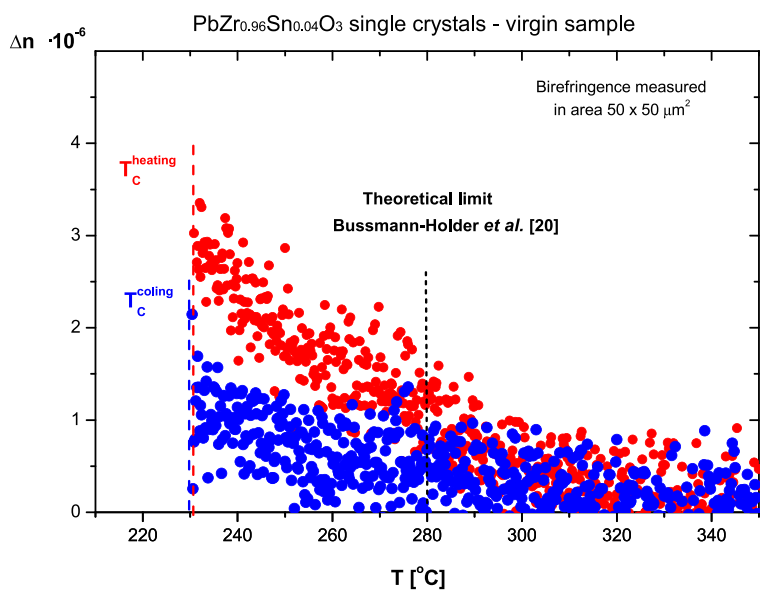

FIG. 2. Thermal hysteresis of birefringence $\Delta n$ in the paraelectric phase. Red and blue points represent points on heating and cooling, respectively. 


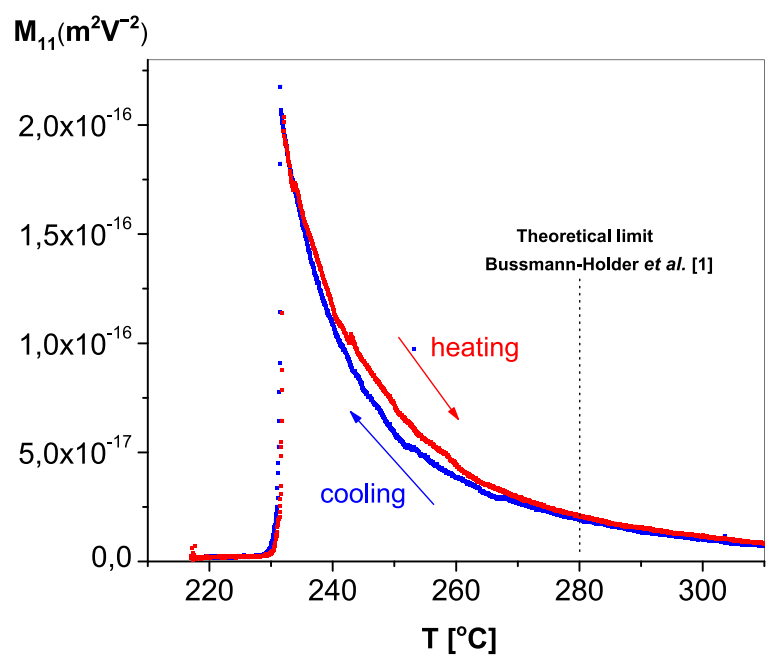

FIG. 3. Electrostrictive coefficient $M_{11}$ as a function of temperature for $\mathrm{PbZr}_{0.96} \mathrm{Sn}_{0,04} \mathrm{O}_{3}$ single crystal. An alternating strain of $320 \mathrm{~Hz}$ was induced by an ac electric field with $E_{\sim}=3 \mathrm{kV} \mathrm{cm}{ }^{-1}$ and frequency $160 \mathrm{~Hz}$. Distinct thermal hysteresis in electrostrictive strain is clearly seen.

previous observations for barium titanate, ${ }^{19}$ in considered temperature range above $\mathrm{T}_{\mathrm{C}}$, there was a lack of macroscopic piezoelectric activity in the investigated PZS crystal.

To check this unexpected result, we have searched for local (nano) piezo- and electrostrictive signals in single PZS crystal by means of PEFM method. We are aware that PEFM is classified as a method exploring properties of surface, but in fact it gives experimental signals, which contain information whether about properties of surface or bulk separately, or are mixture of them.

\section{THE MEASUREMENTS IN NANOSCALE}

All PEFM measurements were done on a modified Jeol 4210 AFM fitted with the commercially available heating element. To facilitate desorption, crystal was heated and cooled under high vacuum $\left(3 \times 10^{-5}\right.$ mbar $)$ conditions. A voltage of $U_{\mathrm{pp}}=5 \mathrm{~V}$ and frequency $f=6.2 \mathrm{kHz}$ was applied to the PtIr coated cantilever ("ContPt" from Nanosensors, $f_{\mathrm{o}}=13 \mathrm{kHz}$ ). The piezoelectric response was detected as the first harmonic component of the deflection of the AFM probe cantilever. Simultaneously to the piezoresponse measurement, the local electrostrictive response was also measured as the second harmonic of the tip displacement. The aim of the measurements was determination the piezo- and electrostrictive activity over a $10 * 10 \mu \mathrm{m}^{2}$ area of a crystal surface. Size of crystal was of the order of $5 \times 5 \times 0.5 \mathrm{~mm}^{3}$. The piezoelectric and electrostrictive activity of the scan was calculated as average value of the absolute value of each measurement point. To get the temperature dependences of PEFM signals were detected in the temperature range $140-300^{\circ} \mathrm{C}$.

Figures 4 and 5 show piezo- and electrostrictive response images acquired in the antiferroelectric (a) and paraelectric ((b) and (c)) phase, respectively, on the first and second harmonic of the tip displacements. All of the images are presented for the same region of crystal surface. In the antiferroelectric phase, piezoelectric signal has not been detected (signal shown in Fig. 5(a) below $\mathrm{T}_{\mathrm{c}}$ is a setup

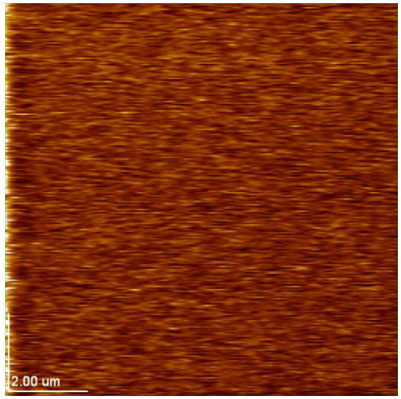

(a)

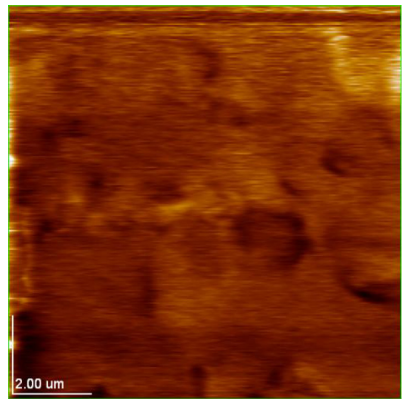

(b)

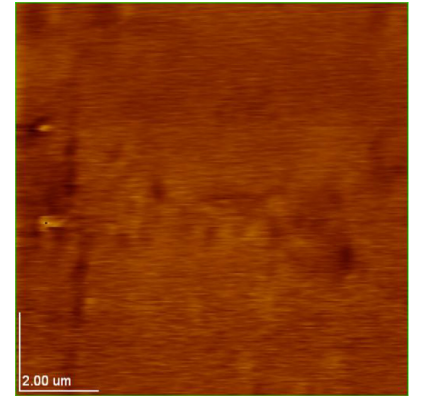

(c)

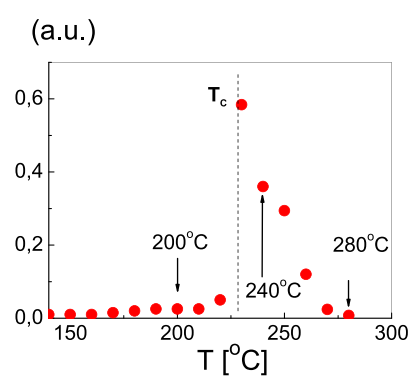

(d)
FIG. 4. Out of plane PFM images $\left(10 * 10 \mu \mathrm{m}^{2}\right)$ for $\mathrm{PbZr}_{0.96} \mathrm{Sn}_{0.04} \mathrm{O}_{3}$ single crystal acquired at A-phase (a), P-phase (b), and deep in P-phase (c). Figure (d) represents temperature dependence of the local piezoelectric response. Below $T_{\mathrm{c}}$ experimental points represents a background.

noise). Assuming that this phase is as that in pure lead zirconate, which is Pbam and thus centrosymmetric, this result is understandable. The fact that electrostrictive activity was not found means that external field was not strong enough to excite measureable deformation. The bright and dark areas visible in the paraelectric phase correspond to regions with

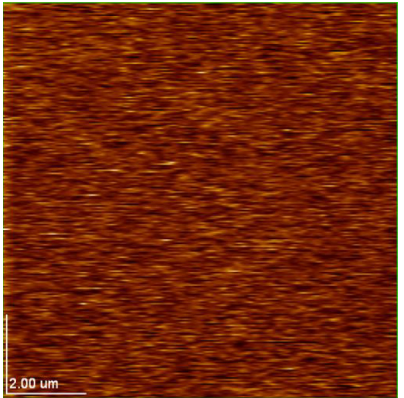

(a)

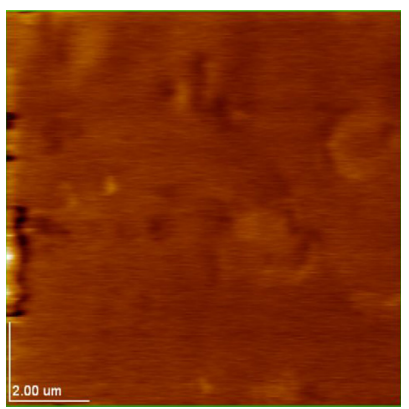

(b)

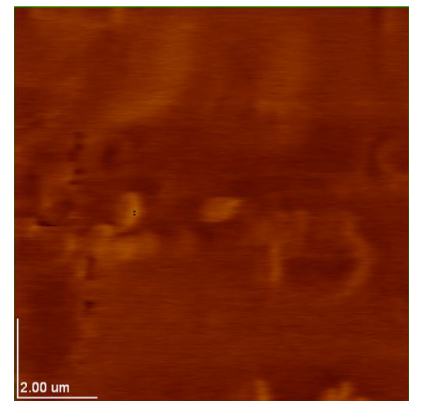

(c)

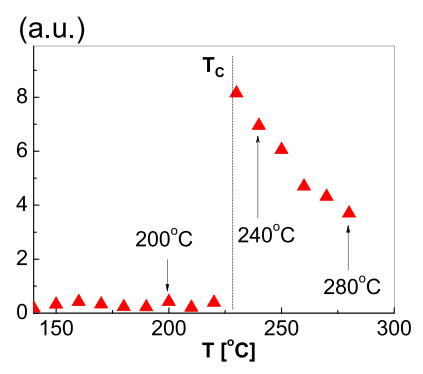

(d)
FIG. 5. Out of plane EFM images $\left(10 * 10 \mu \mathrm{m}^{2}\right)$ for $\mathrm{PbZr}_{0.96} \mathrm{Sn}_{0.04} \mathrm{O}_{3}$ single crystal at A-phase (a), P-phase (b), and deep in P-phase (c). (d) represents temperature dependence of the local electrostrictive response. 
the polarization oriented up and down relative to the surface level. An intermediate contrast means that piezoresponse is negligible.

Qualitatively, the same dependences have been recognized for the piezo- and electrostrictive effects measured in plane and are not presented here.

As it was shown in Figures 2 and 3 , above $T_{\mathrm{C}}$, an unexpected thermal hysteresis of the electrostrictive coefficient $M_{11}$ and birefringence has been observed. It is worthwhile to mention here that such hysteresis has also been found in single $\mathrm{BaTiO}_{3}$ and $\mathrm{SrTiO}_{3}$ crystals. ${ }^{18,21}$ Thus, it was interesting to check if similar behavior takes place in crystals with antiferroelectric state. In the case of $\mathrm{BaTiO}_{3}$ and $\mathrm{SrTiO}_{3}$, type of transition is, respectively, of polar ferroelectric and nonpolar antidistortive character.

For PZS crystal under investigation, comparison of the piezoelectric deformation at defined temperature from the paraelectric phase, approached from the high and then from the low temperature side, revealed indeed different values. It is seen in Figures 6(a) and 6(b) where two images of piezoelectric activity were made at $240^{\circ} \mathrm{C}$. Much more inhomogeneous distribution of values of out of plane piezoelectric signals shows image taken at $240^{\circ} \mathrm{C}$ when reached on heating. This distinct difference between these two images indicates existence of thermal hysteresis as that shown in Figure 3. Figure 6(c) presents this hysteresis between $T_{\mathrm{C}}$ and $280{ }^{\circ} \mathrm{C}$. Attention should be paid to the fact that values of piezoelectric effect are higher on heating than on cooling.

Moreover, one can conclude that the observed piezoelectric nanoregions are randomly distributed and boundaries between them are diffused and non-piezoactive. While the piezoelectric effect disappears close to $280^{\circ} \mathrm{C}$, i.e., size of the regions are too small to be detected, the electrostrictive effect, as natural effect for any phase symmetry, is present as far as the sensitivity of the measurement method allows observation. To summarize the experimental findings, one may say that both the bulk and surface properties distinguish the existence of quite broad temperature region $T_{\mathrm{C}}<T<280^{\circ} \mathrm{C}$, in which non-classical behavior of paraelectric phase in PZS crystal has been observed. The wideness of this region is in good agreement with precursor dynamics above $T_{\mathrm{C}}$ recently considered theoretically by Bussmann-Holder et al. ${ }^{1}$

\section{DISCUSSION}

The existence of local and stable non-symmetrical regions (polar clusters) above $T_{C}$ in ferroelectric materials has already been reported. ${ }^{19}$ Those polar clusters (precursor domains) are an intrinsic effect based on the short wavelength structural instability that is related to the long wavelength polar soft mode and driven by the temperature dependent fluctuating dipole moments. ${ }^{1}$ In the case of investigated oxidic perovskite PZS crystal, in which we have to deal alone with the antiferroelectric transformation, such instability cannot be excluded. If so, the piezoactive regions above the phase transition are likely to appear, although experiments described here cannot finally resolve whether these regions are polar or not. The experimental fact is that

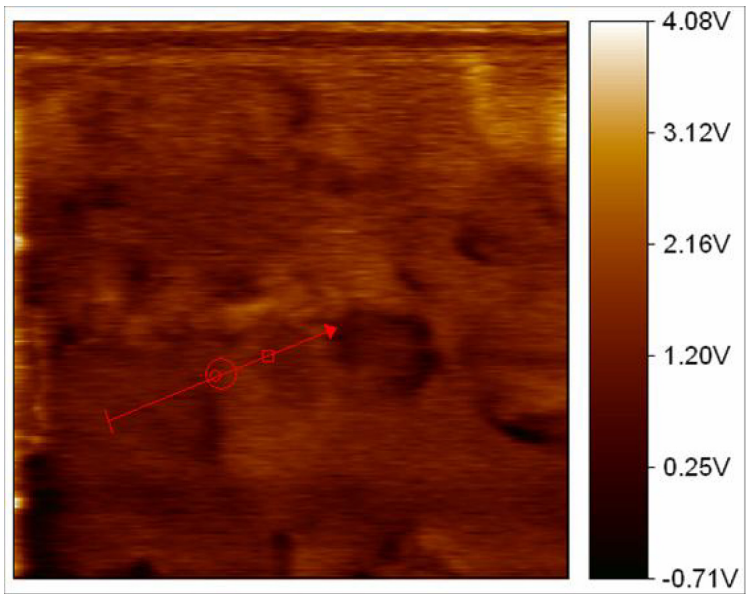

(a)

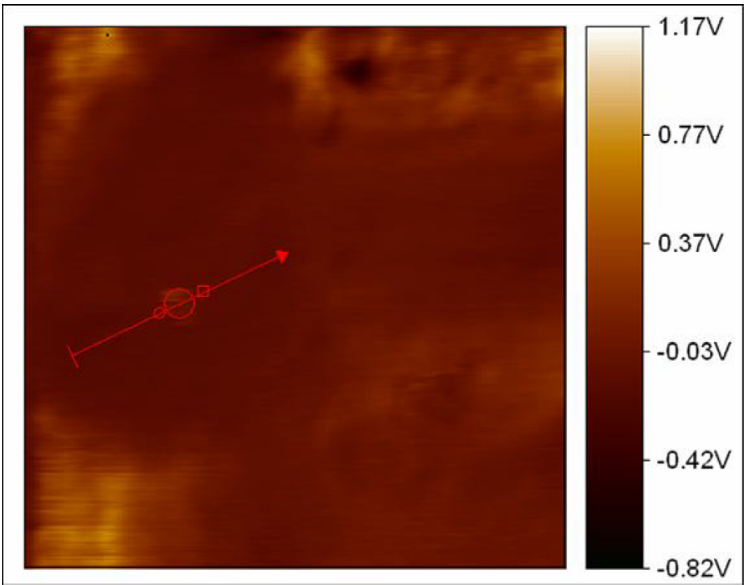

(b)

Piezoresponse out of plane

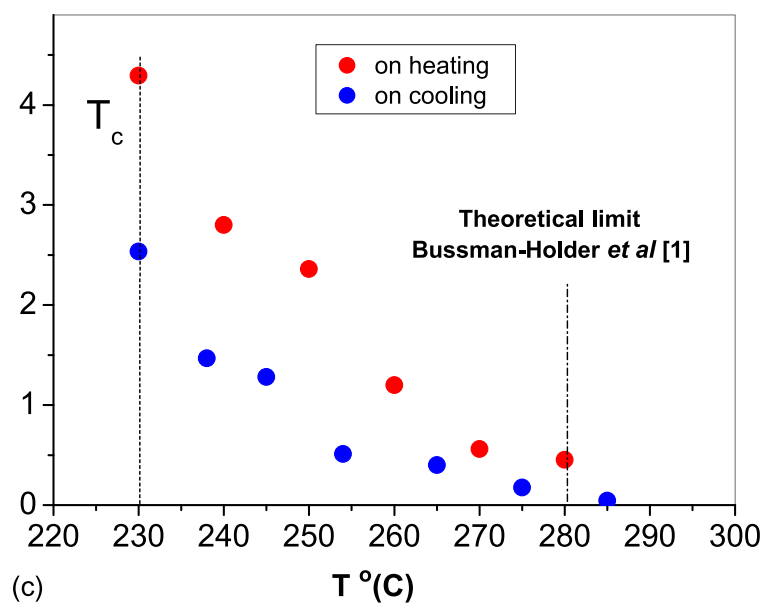

FIG. 6. Comparison of normalized images for the out-of plane piezoresponse measured at $240{ }^{\circ} \mathrm{C}$ reached (a) on heating and (b) on cooling. (c) presents thermal hysteresis in mean value of piezoelectric activity calculated for $10 \times 10 \mu \mathrm{m}^{2}$ area.

below $280^{\circ} \mathrm{C}$ in the investigated PZS crystal, at least part of these regions is stable; otherwise, the static piezoelectric deformation would not be possible to occur at all. On the other hand, taking into account the thermal hysteresis found, it can be conceivable that the local instabilities above $T_{\mathrm{C}}$ might be connected with nonhomogenous distribution of Sn ions in 
crystal lattice substituting $\mathrm{Zr}$ ions centrally placed in the centre of oxygen octahedron. Though it is difficult to believe that such distribution changes passing phase transitions, it can differently act on local phase transition evolution on cooling and heating.

What is not theoretically predicted, and as far as we know even not discussed in literature, is the thermal hysteresis of physical properties in the paraelectric phase of $\mathrm{ABO}_{3}$ perovskites, similar to that we have described in this report. From theory, ${ }^{1}$ it is derived for ferroelectric perovskites that the lattice inhomogeneity as clusters or precursor domains above $T_{\mathrm{C}}$ is an intrinsic effect based on the short wavelength structural instability that is related to the long wavelength polar soft mode and driven by the temperature dependent fluctuating dipole moment. The temperature evolution of this interdependence of the structural and polar soft mode could be responsible for non-classical dependences reported earlier. $^{22}$

Similar mechanism can be applicable to PZS. The question remains as to why both in the case of ferroelectric and antiferroelectric transitions this thermal hysteresis takes place. Most probably, the common mechanism is connected with size evolution of clusters or precursor domains above $T_{\mathrm{C}}$. Rejuvenation at high temperatures, of the order of $400{ }^{\circ} \mathrm{C}$, may already change distribution of point defects in oxygen sublattice, making them homogenously distributed on the way to phase transition point from the high temperature side. In opposite, the distribution of these defects in phase below $T_{\mathrm{C}}$, where they may be mainly accumulated in domain boundaries, is strongly inhomogeneous. When phase transition is approached from the low temperature phase, domain boundaries disappear, leaving crystal lattice with local strains produced by inhomogeneous oxygen defects distribution.

Another possibility is influence of stacking faults, the existence of which has been revealed in $\mathrm{SrTiO}_{3}{ }^{23}$ and mainly related to oxygen vacancies arising during crystal growth, which may break the cubic symmetry statically. On the other hand, high-resolution transmission electron microscopy, $\mathrm{x}$-ray photoelectron spectroscopy, or atomic force microscopy have provided evidence that dislocations and shear planes are also present in perovskites. Around these lattice faults, which are difficult to remove by thermal rejuvenation, the local mechanical and electrical stresses are created. These stresses may eventually cause the thermal hysteresis appearance. Especially, the dislocations, very mobile in oxidic perovskite, may play essential role because their distribution in the bulk may change while passing phase transition point. Theoretical models considering such mechanism would be interesting to develop.

In this place, we may conclude that thermal hysteresis and mechanism discussed above could be of general character and thus applied to pure lead zirconate too. As mentioned in the Introduction, between the AFE and PE structures, an intermediate ferroelectric phase of rhombohedral symmetry may appear. ${ }^{8-11}$ In fact, in samples of pure lead zirconate crystal in which such intermediate phase was verified beyond doubt, symptoms of similar thermal hysteresis of $\Delta n(\mathrm{~T})$ as that observed in PZS has been observed.

\section{ACKNOWLEDGMENTS}

This research was financially supported by the NCN within the project 1955/B/H03/2011/40. Partial support within the project SFB917 is also kindly acknowledged.

\footnotetext{
${ }^{1}$ A. Bussmann-Holder, H. Beige, and G. Völkel, Phys. Rev. B 79, 184111 (2009).

${ }^{2}$ M. E. Lines and A. M. Glass, Principles and Applications of Ferroelectrics (Clarendon Press, Oxford, 1977).

${ }^{3}$ G. Kugel, I. Jankowska-Sumara, K. Roleder, and J. Dec, J. Korean Phys. Soc. 32, S581 (1998).

${ }^{4}$ X. Dai, J.-F. Li, and D. Viehland, Phys. Rev. B 51, 2651 (1995).

${ }^{5}$ S. Roberts, J. Am. Ceram. Soc. 33, 63 (1950).

${ }^{6}$ G. Shirane, E. Sawaguchi, and A. Takeda, Phys. Rev. 80, 485 (1950).

${ }^{7}$ G. Shirane, E. Sawaguchi, and Y. Takagi, Phys. Rev. 84, 476 (1951).

${ }^{8}$ A. E. Pasto and S. R. A. Condrate, J. Am. Ceram. Soc. 56, 436 (1973).

${ }^{9}$ H. Liu and B. Dkhil, Z. Kristallogr. - Crystal. Mater. 226, 163-170 (2011).

${ }^{10}$ Z. Xu, X. Dai, D. Viehland, D. A. Payne, Z. Li, and Y. Jiang, J. Am. Ceram. Soc. 78, 2220 (1995).

${ }^{11}$ D. Viehland, Phys. Rev. B 52, 778 (1995).

${ }^{12}$ K. Roleder and J. Dec, J. Phys.: Condens. Matter 1, 1503 (1989).

${ }^{13}$ S. Wirunchit, P. Laoratanakul, and N. Vittayakorn, J. Phys. D: Appl. Phys. 41, 125406 (2008).

${ }^{14}$ I. Jankowska-Sumara, A. Majchrowski, and J. Zmija, J. Mater. Sci. 44, 3229 (2009)

${ }^{15}$ I. Jankowska-Sumara, M. Górny, K. Rusek, A. Majchrowski, J. Żmija, and K. Roleder, Ferroelectrics 400, 89-95 (2010).

${ }^{16}$ I. Jankowska-Sumara, A. Majchrowski, and J. Żmija, Phase Trans.84, 521-527 (2011).

${ }^{17}$ M. A. Geday, W. Kaminsky, J. G. Lewis, and A. M. Glazer, J. Microsc. 198, 1-9 (2000)

${ }^{18}$ A. Ziębińska, D. Rytz, K. Szot, M. Górny, and K. Roleder, J. Phys.: Condens. Matter 20, 142202 (2008)

${ }^{19}$ K. Rusek, J. Kruczek, K. Szot, D. Rytz, M. Górny, and K. Roleder, Ferroelectrics 375, 165-169 (2008).

${ }^{20}$ K. Roleder, J. Phys. E: J. Sci. Instrum. 16, 1157 (1983).

${ }^{21}$ K. Roleder, A. Bussmann-Holder, M. Górny, K. Szot, and A. M. Glazer, Phase Trans. 85, 939-948 (2012).

${ }^{22}$ J.-H. Ko, T. H. Kim, K. Roleder, and S. Kojima, Phys. Rev. B 84, 094123 (2011).

${ }^{23}$ K. Szot, W. Speier, G. Bihlmayer, and R. Waser, Nature Mater.5, 312 (2006).
} 